\title{
LA FORMULACIÓN Y EVALUAACIÓN \\ DE PROYECTOS DE \\ INVERSIÓN: UNA NUEVA \\ VISIÓN PARA LAS PYMES \\ DE SERVICIO
}

\section{RESUMEN}

El objetivo de este artículo es presentar una nueva visión de la Formulación y Evaluación de Proyectos de Inversión, proponiendo un marco comprehensivo para las pequeñas y medianas empresas del sector de servicios en la ciudad de Bogotá, que permita a los empresarios entender y obtener mejores desempeños en sus proyectos. El marco propuesto está basado en la Teoría de Recursos y Capacidades, y enmarcado en las capacidades de gestión, generación, transferencia y la utilización del conocimiento, y establecimiento de un proceso de toma de decisión. El método tiene como base una investigación no experimental, de corte transversal, en donde se verifica el modelo de manera empírica y causal, utilizando la Regresión Categórica.

Palabras clave: Formulación y evaluación de proyectos de inversión; Teoría de los recursos y capacidades; Gestión de proyectos; Información y conocimiento; Proceso de toma de decisión; Desempeños en proyectos. 


\section{FORMULATION AND EVALUATION OF INVESTMENT PROJECTS. A NEW VISION FOR SMES IN THE SERVICES SECTOR}

\section{ABSTRACT}

The aim of this paper is to present a new vision of Formulation and Evaluation of Investment Projects, proposing a comprehensive framework for small and medium enterprises in the services sector in the city of Bogotá, which allows businessman to understand and better performance in their projects. The proposed framework is based on the Theory of Resources and Capabilities, and framed management capabilities, generation, transfer and use of knowledge, and establishment of a decision-making process. The method is based on a non-experimental research, cross-sectional, where the empirical model and causally is verified using the Categorical Regression.

Keywords: Formulation and Evaluation of Investment Projects; Theory of Resources and Capabilities; Project Management; Information and Knowledge; Decision Making Process; Projects Performance.

\section{INTRODUCCIÓN}

Una de las tareas más significativas de la gerencia lo constituye el hecho de tomar decisio- nes acerca de la asignación de recursos para los nuevos proyectos (Adler, 2000; Baldwin y Clark, 1992, 1994; Bower, 1995; Maritan, 2001; Wheelwright, 1992). En el mercado, las empresas compiten y desean ganar; este propósito las mantiene alertas. No obstante, y para que este no se pierda, deben estar soportadas en una acción que implica esfuerzo; este propósito es el que conduce la asignación de recursos mediante la formulación y evaluación de nuevas estrategias, enfatizando que es traer el futuro al presente. "El propósito estratégico implica un esfuerzo considerable para una organización" (Hamel y Prahalad, 1995, p. 96). "Las inversiones que comprometen los recursos de la empresa a largo plazo son inversiones estratégicas" (Ketelhohn, Marín, y Montiel, 2004, p. 4); así, la estrategia ha sido definida como "la unión que hace una organización entre los recursos y habilidades internas y la oportunidad y el riesgo creado por el ambiente externo" (Grant, 1991, p. 114).

Los modelos de presupuestos de capital son los métodos que las empresas utilizan para decidir cómo se evalúan y asignan los recursos de inversión entre los proyectos, teniendo un gran impacto sobre el resultado de las empresas y las economías (Baldwin y Clark, 1992, 1994; Porter, 1992); los recursos, como las personas y el conocimientos son comprometidos en proyectos buscando un retorno esperado futuro que supere el costo de oportunidad (Maritan, 2001). 
Para ello, las empresas formulan y evalúan diferentes alternativas sobre las cuales toman decisiones, estableciendo su posible ejecución o no; buscando con ello encontrar la decisión óptima de inversión, es decir, aquellas que maximizan el valor presente de la riqueza de los accionistas (Copeland y Weston, 1992). Sin embargo, los empresarios no necesariamente toman decisiones óptimas; por el contrario, se ajustan a lo que ellos consideran como mejor, dentro del análisis conocido como la racionalidad acotada o limitada (Simon, 1972), y muy particularmente los dueños de las pequeñas y medianas empresas - PYMES, que poseen una serie de características propias que los dife- rencian en sus análisis de los realizados por las empresas grandes, en donde "las pequeñas empresas no comparten los mismos problemas de gestión financiera con las grandes empresas" (Ang, 1991, p. 1).

Las características expresadas en la tabla 1 llevan a que la Formulación y Evaluación de Proyectos de Inversión (FYEPI), la herramienta fundamental para definir los nuevos proyectos, se vea enfrentada en las PYMES a una serie de hechos particulares, como la ausencia de capacidades gerenciales para propiciar y generar este tipo de análisis, la deficiencia en la información relevante acerca de los proyectos y la falta de un proceso de toma de decisión.

Tabla 1. Características únicas de las PYMES con relación a las grandes empresas

\begin{tabular}{|c|c|}
\hline No cotiza en la bolsa de valores. & $\begin{array}{l}\text { No se tiene una valoración al día, la información de la empresa del mercado con } \\
\text { relación a la empresa es muy baja y tiene dificultad para acceder al mercado } \\
\text { financiero con menores costos. }\end{array}$ \\
\hline $\begin{array}{l}\text { Los propietarios no diversifican sus } \\
\text { carteras personales. }\end{array}$ & El patrimonio de los dueños está, en un alto porcentaje, vinculado con la empresa. \\
\hline $\begin{array}{l}\text { La responsabilidad limitada se } \\
\text { encuentra ausente o es ineficaz. }\end{array}$ & $\begin{array}{l}\text { En ocasiones los acreedores obligan de manera contractual a los dueños a } \\
\text { proporcionar garantías personales como garantía para préstamos. }\end{array}$ \\
\hline $\begin{array}{l}\text { Propietarios de primera generación son } \\
\text { emprendedores y propensos a tomar } \\
\text { riesgos. }\end{array}$ & $\begin{array}{l}\text { Los fundadores de la empresa son personas visionarias y ambiciosas que realizan } \\
\text { un gran esfuerzo, el cual se ve compensado con unos altos rendimientos; sin } \\
\text { embargo, esto genera altos niveles de riesgo. }\end{array}$ \\
\hline El equipo de gestión no está completo. & $\begin{array}{l}\text { Se depende de una sola persona para tomar la mayoría de las decisiones, y ante } \\
\text { la ausencia de esta persona, la empresa simplemente queda huérfana. Presentan } \\
\text { deficiencias para armar equipos multidisciplinarios. }\end{array}$ \\
\hline $\begin{array}{l}\text { Por imperfecciones en el mercado } \\
\text { pagan altos costos de quiebra y } \\
\text { liquidación. }\end{array}$ & $\begin{array}{l}\text { LaS PYMES son propensas a pagar proporcionalmente más costos en escenarios de } \\
\text { quiebra y liquidación. }\end{array}$ \\
\hline $\begin{array}{l}\text { Las relaciones con los accionistas son } \\
\text { menos formales. }\end{array}$ & $\begin{array}{l}\text { Al ser pocos los accionistas gestores de la empresa y, en algunos casos, como el } \\
\text { de las empresas de familia, existen vínculos entre ellos, la comunicación es más } \\
\text { personal y menos contractual. }\end{array}$ \\
\hline
\end{tabular}

Fuente: Ang (1991).

LaS PYMES constituyen uno de los grandes eslabones en las economías. Su importancia radica, además de su participación en el total del número de empresas, en los aportes en el empleo y en el producto interno bruto de los países (Ferrero y Stumpo, 2010).

De acuerdo con la OCDE y la CEPAL, un aspecto fundamental de las PYMES latinoame- ricanas es su extrema heterogeneidad porque mientras en algunas su "gestación suele responder a necesidades individuales de autoempleo, y que a menudo están en una situación de informalidad", otras, por el contrario, con alto crecimiento, son "conocidas como "gacelas", que tienen un comportamiento bastante más dinámico en materia de facturación y creación 
de puestos de trabajo y cuyo desempeño responde al aprovechamiento de oportunidades de mercado a través de una gestión empresarial eficiente e innovadora (2012, p. 48).

De acuerdo con Bello (2013):

La mayoría de las PYMES colombianas, por las condiciones estructurales de su creación, dependen de la experiencia de sus dueños, su manejo es centralizado, generando un control de información que no trasciende y que impide que haya empoderamiento por parte de las personas que laboran al interior, esto se traduce en problemas como calidad deficiente en los productos y servicios, demora en las entregas, ciclos de producción o de operación deficientes, baja eficiencia en los usos de recursos, baja productividad, etc. (p. 13).

En Colombia, las PYMES "ofrecen el 57\% del empleo y representan el $30 \%$ de las ventas totales" (AcoPI, 2006, p. 5); "en general, las PYMES están presentes en todos los sectores de la economía, siendo más fuerte su presencia en el sector comercio" (Ferrero y Stumpo, 2010, p. 19). Según el Departamento Nacional de Estadísticas - DANE, para el año 2005 se reportaron 1.442 .117 empresas, de las cuales el $93 \%$ son microempresas y $3,50 \%$ son PYMES, es decir, 50.474 (MINCIT, 2014). De acuerdo con el Banco Mundial, las PYMEs emplean alrededor del $30 \%$ de la fuerza laboral y, de ese total, el sector servicios emplea el 59\% (2008).

En Colombia, las PYMEs están determinadas por la Ley 905 de 2004, la cual modificó la Ley 590 de 2000. Se considera una PYME a aquella unidad de explotación económica que responda por lo menos a alguno de los dos parámetros de clasificación, como es el número de trabajadores y el total de activos. La pequeña empresa cuenta con entre 11 y 50 empleados y activos superiores a 500 SMLMV $^{1}$ y hasta los 5.000. De otro lado, la mediana empresa cuenta con entre 51 y 200 empleados y activos superiores a 5.000 SMLMV y hasta los 30.000 .

El objetivo del presente artículo es mostrar una nueva visión de la Formulación y Evaluación de Proyectos de Inversión, proponiendo un modelo comprehensivo para las pequeñas y medianas empresas en la ciudad de Bogotá, del sector de servicios, que permita a los empresarios entender y obtener mejores desempeños en sus proyectos; todo esto basado en la Teoría de Recursos y Capacidades, y enmarcado en las capacidades de gestión, de generación, transferencia y la utilización del conocimiento, y establecimiento de un proceso de toma de decisión.

\section{MARCO TEÓRICO}

De acuerdo con su definición, formular consiste en reducir a términos claros y precisos una proposición o expresar algo por medio de una fórmula; y evaluar, consiste en señalar valor a una cosa, siendo igualmente estimar o apreciar (RAE, 2001). Ahora, la palabra proyecto viene del latín proiectus (RAE, 2001), que significa lanzar hacia adelante, o hacia el futuro (Etimologías, s.f.). Finalmente, la inversión corresponde a la acción y efecto de emplear, gastar, ocupar un caudal extendido en el tiempo, por lo que "inversión significa formación de capital" (Ketelhohn, Marín, y Montiel, 2004, p. 27), para las "empresas invertir es una cuestión de supervivencia en un contexto de economías abiertas y en competencia" (López, 1995).

Según el ILPES, los proyectos "son cursos de acción que se originan a partir de un problema y dan respuesta a ese problema en 
forma racional. Esto implica el uso eficiente de los recursos disponibles" (Ortegón, Pacheco, y Roura, 2005, p. 11).

Así, al involucrar el tema empresarial, se puede decir que una empresa formula y evalúa sus proyectos de inversión cuando define en términos claros y precisos una acción futura, la cual valora de forma cuantitativa buscando obtener futuros beneficios superiores al esfuerzo de la inversión inicialmente ejecutada, actuando en escenarios de incertidumbre. Sin embargo, esto corresponde al hecho de que la acción requiere de un "gatillo" que la inicie, una necesidad u oportunidad presente en el mercado, para las cuales las empresas están atentas. Los proyectos se generan por una necesidad de los consumidores (Rosillo, 2008, p. 2). A partir de estas necesidades $u$ oportunidades, las empresas definen una idea que desean cristalizar, es decir, formulan y evalúan sus actuaciones futuras, que definen como proyectos de inversión. Por último, para el desarrollo de análisis de la formulación y evaluación, se requiere contar con el conocimiento, las habilidades y la motivación del recurso humano.

\subsection{ALGUNAS DEFINICIONES DE LA FYEPI}

Para Araújo, la formulación y evaluación tiene la "finalidad de generar, evaluar, comparar y seleccionar alternativas de inversión, eligiendo la más eficiente, para satisfacer una necesidad específica" (Araújo, 2012, p. 15).

Para Mokate, la formulación consiste en explorar "diversos caminos potenciales para satisfacer una determinada necesidad" (Mokate, 2007, p. 21), y realiza una crítica a la formulación, ya que por la facilidad de las comunicaciones, los estudios exitosos hoy en día, simplemente son replicados, convirtiéndolos en meras "recetas" para volver la formulación en "estudios de optimización de los pormenores técnicos y en una sobreespecificación de los detalles operativos de la iniciativa" (Mokate, 2007, p. 22), proponiendo que la formulación de proyectos vuelva a su razón de ser: estudiar el entorno que ellos afectarán y transformarán.

Para Infante (1988), los proyectos de inversión cuantifican los ingresos y egresos que se hacen con base en sumas de dinero que el dueño del proyecto recibe, entrega o deja de recibir de las diferentes alternativas, siendo un análisis microeconómico en donde priman los precios del mercado. Igualmente, menciona que se pueden definir la formulación y la evaluación para proyectos del ámbito económico y social.

\subsection{CLASIFICACIÓN DE LOS PROYECTOS}

Como resumen de la tabla 2, encontramos que los proyectos de inversión se pueden dividir en aquellos que son emprendidos de ceros, es decir, el proyecto da origen a una nueva empresa, la cual está vinculada normalmente con el desarrollo de nuevos productos y los proyectos que desarrollan en las empresas que ya se encuentran en marcha, dirigidos al mejoramiento de la eficiencia en los costos, establecer reemplazos de la maquinaria y equipos existentes, los enfocados a externalizar o internalizar actividades o procesos, los dirigidos a nuevos productos y a la conquista de nuevos mercados. 
Tabla 2. Algunas clasificaciones de los proyectos

\begin{tabular}{|c|c|}
\hline Autor & Componentes de la clasificación \\
\hline Joel Dean (1973) & a) Renovación, b) modernización, c) expansión y d) estratégicos. \\
\hline Joseph L. Bower (1995) & $\begin{array}{l}\text { a) Los correspondientes a la reducción de costos, b) los que buscan nuevos mercados } \\
\text { y c) los que buscan el desarrollo de nuevos productos. }\end{array}$ \\
\hline James C. Van Horne (1997) & $\begin{array}{l}\text { a) Nuevos productos o expansión de productos ya existentes, b) reemplazo de } \\
\text { equipos o edificios, c) investigación y desarrollo, d) exploración y e) otros. }\end{array}$ \\
\hline Nassir Sapag (2001). & a) Reemplazo, b) ampliación, c) externalización, d) internalización y e) abandono. \\
\hline $\begin{array}{l}\text { A Guide to the Project Management } \\
\text { Body of Knowledge (Institute, Project } \\
\text { Management, 2013) }\end{array}$ & $\begin{array}{l}\text { a) Demanda del mercado, b) necesidades organizacionales, c) solicitudes de los } \\
\text { clientes, d) avances tecnológicos, e) requerimientos legales, f) disminución del } \\
\text { impacto ecológico y g) necesidades sociales. }\end{array}$ \\
\hline $\begin{array}{l}\text { Crawford, L., Hobbs, B. y Turner, J. R. } \\
\text { (2005) }\end{array}$ & $\begin{array}{l}\text { a) Área de aplicación, b) complejidad, c) importancia estratégica, d) tipo de } \\
\text { contratación, e) etapa del ciclo de vida y f) cultura. }\end{array}$ \\
\hline Larson, E.W. y Gray, C. F. (2011) & a) Proyectos de cumplimiento, b) operacionales y c) estratégicos. \\
\hline
\end{tabular}

Fuente: Elaborada por el autor con base en Dean (1973), Bower (1995), Van Horne (1997), Sapag (2001), PMI (2013), Crawford, Hobbs y Turner (2005) y Larson y Gray (2011).

Morris et al. (2010) han definido lo anterior, como una clasificación desde el punto de vista del emprendimiento, en donde se presenta una iniciativa empresarial de capital riesgo o un emprendimiento estratégico, lo que constituye el hecho de crear la empresa desde el inicio para aprovechar la oportunidad de mercado, 0 corresponde a la búsqueda de oportunidades y ventajas competitivas de las empresas ya creadas (Arzubiaga, Iturralde y Maseda, 2012).

\subsection{ETAPAS DEL PROYECTO}

Los proyectos de inversión en su desarrollo presentan unas etapas claramente definidas y secuenciales (ver gráfico 1): idea, preinversión, inversión, operación y, en algunos casos, abandono o liquidación (Sapag y Sapag, 2008).

Gráfico 1. Etapas del ciclo de vida del proyecto

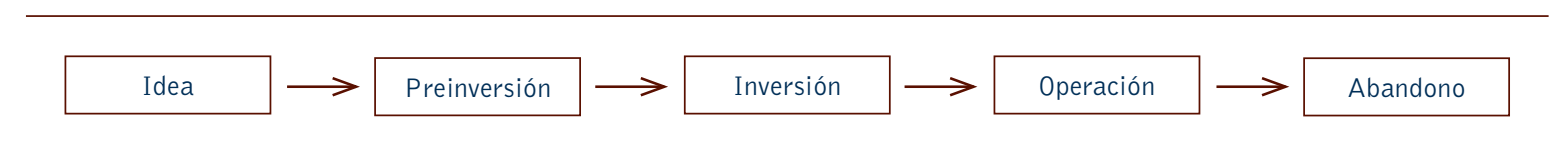

Fuente: Sapag y Sapag (2008).

La etapa en donde se ubican la formulación y la evaluación corresponde a la preinversión ${ }^{2}$; esto da como resultado la viabilidad del proyecto, y si el proyecto de inversión es viable, se procede a implementarlo en la etapa de inversión.
De acuerdo con el gráfico 2, la etapa de preinversión presenta tres estudios: perfil, prefactibilidad y factibilidad (Sapag y Sapag, 2008; Baca, 2006).

En el perfil, se busca un primer acercamiento a la realización del proyecto, soportado

2 En algunas ocasiones, a la formulación y evaluación se las conoce como estudios de preinversión. 
en información obtenida de estudios generales del sector, algunos comentarios de personas del medio y entrevistas con expertos en el área en que se desarrollará el proyecto. En este se determina si existen variables de peso que determinen la no realización del proyecto (Sapag y Sapag, 2008).

Gráfico 2. Etapas de la preinversión

Perfil $\rightarrow$ Prefactibilidad $\rightarrow$ Factibilidad

Fuente: Sapag y Sapag (2008).

En la prefactibilidad, también Ilamada anteproyecto (Baca, Evaluación de Proyectos - Análisis y Administración de Riesgo, 1990), se busca profundizar la información de las diferentes alternativas, trabajando con fuentes de información secundaria, es decir, aquellas que han sido desarrolladas para otros propósitos (Kotler, 1999). En este nivel, podemos medir la rentabilidad de las posibles soluciones y descartar alternativas, dejando una en lo posible. Con la información recogida en el nivel de prefactibilidad, se define una única alternativa, recomendando la continuación del estudio si es necesario, o simplemente su abandono o postergación.

En el nivel de factibilidad, los estudios se realizan con fuentes de información primaria, de manera que todas las variables se expresen de forma cuantitativa y no cualitativa. La información primaria es elaborada especialmente para el proyecto, de allí su alto costo.

Los estudios de prefactibilidad y factibilidad son soportados por una serie de estudios agrupados dependiendo del origen de la información, estos son conocidos como estudios de mercado, técnico o de producción, de gestión, legal, ambiental, social y financiero (Sapag y Sapag, 2008).

Cada uno de los diferentes estudios da respuesta a una serie de interrogantes que se definen para garantizar la factibilidad del pro- yecto. Así, el estudio de mercado establece la oportunidad en el mercado y su demanda, analiza la competencia y define cómo llegar al cliente, en lo que se conoce como la estrategia comercial.

El estudio técnico o de producción revisa las consideraciones relevantes al diseño del producto, el proceso productivo, la capacidad, la localización y sus costos. El estudio de gestión define la planeación estratégica, y a partir de ella establece la estructura organizacional. El estudio legal precisa los aspectos jurídicos que impactan el proyecto, los temas de constitución de la nueva empresa, si es el caso, y los permisos, licencias y trámites necesarios para su operación.

Los estudios ambiental y social revisan los impactos positivos y negativos que la realización del proyecto va a tener en su ámbito y en la comunidad. Y, finalmente, el estudio financiero revisa temas como los presupuestos de ingresos, costos, gastos e inversión, la financiación y sus costos y la rentabilidad y generación de valor. Al final, cada uno de los estudios presenta su propia factibilidad.

\subsection{MARCO PROPUESTO}

El nuevo marco propuesto para la Formulación y Evaluación de Proyectos de Inversión tiene un enfoque sistémico soportado en la teoría basada en recursos y capacidades, y en donde se espera como resultado entender y obtener mejores desempeños en los proyectos.

\subsubsection{RECURSOS Y CAPACIDADES}

La teoría parte de las fortalezas en los recursos y capacidades particulares de la empresa que la llevan a tener un buen desempeño y ventajas competitivas sostenibles en el tiempo (Wernerfelt, 1984; Barney, 1991). Sin embargo, y por 
lo reciente de la teoría (Dávila, 2009), no se presenta un consenso en cuanto a la palabra recursos, teniendo diferentes aproximaciones y definiciones, algunas de las cuales se enuncian en la tabla 3.

Tabla 3. Algunas definiciones de recursos

\begin{tabular}{|c|c|}
\hline Grant (1991) & $\begin{array}{l}\text { Los recursos corresponden a } \\
\text { las unidades de entrada en los } \\
\text { procesos productivos de las } \\
\text { empresas, formando la unidad de } \\
\text { base de análisis. }\end{array}$ \\
\hline $\begin{array}{l}\text { Grant (V.L.y } \\
\text { Crittenden, 2008) }\end{array}$ & $\begin{array}{l}\text { Se pueden clasificar en tangibles, } \\
\text { intangibles y el recurso humano. }\end{array}$ \\
\hline Wernerfelt (1989) & $\begin{array}{l}\text { De acuerdo con su potencial } \\
\text { de uso, los recursos tienen tres } \\
\text { categorías: a) con potencial fijo, } \\
\text { b) con potencial ilimitado y c) } \\
\text { recursos con un potencial fijo a } \\
\text { corto plazo pero ilimitado a largo } \\
\text { plazo. }\end{array}$ \\
\hline Barney (1991) & $\begin{array}{l}\text { Los recursos deben ser valiosos; } \\
\text { deben poseer los atributos clave } \\
\text { de ser raros, imperfectamente } \\
\text { imitables e insustituibles. }\end{array}$ \\
\hline $\begin{array}{l}\text { Dierickx y Cool } \\
\text { (1989) }\end{array}$ & $\begin{array}{l}\text { Los activos particulares son } \\
\text { bienes no transables, no imitables } \\
\text { e insustituibles. }\end{array}$ \\
\hline Itami (1989) & $\begin{array}{l}\text { Los activos estratégicos de una } \\
\text { empresa son invisibles. }\end{array}$ \\
\hline Rumelt (1984) & $\begin{array}{l}\text { Surgen de eventos inesperados } \\
\text { que crean rentas potenciales } \\
\text { en presencia de mecanismos de } \\
\text { aislamiento y tienen ambigüedad } \\
\text { causal y complejidad social. }\end{array}$ \\
\hline
\end{tabular}

Fuente: Elaborada por el autor con base en Grant (1991), Grant (2010), Wernerfelt (1989), Barney (1991), Dierickx y Cool (1989), Itami (1989), Rumelt (1984).

Sin embargo, los recursos por sí solos no son suficientes para obtener una verdadera ventaja competitiva sostenible en el tiempo; para ello, es necesario que se conviertan en capacidades y estas en desempeño positivo para la empresa (Mahoney y Padian, 1992); (Barney, 1991). "Un ingrediente clave en las relaciones entre los recursos y las capacidades es la habilidad de una organización para lograr cooperación y coordinación" (Grant, 1991, p. 122). "Los recursos son medios aislados, necesarios pero no suficientes para alcanzar los fines de la empresa" (Brunet, 2011, p. 152) necesitando de las capacidades para integrar los recursos de diferente naturaleza y poder llevar a cabo actividades productivas (Brunet, 2011).

Las capacidades tienen la consideración de flujo, es decir, representan el aspecto dinámico, siendo las que definen la forma en que la empresa emplea sus recursos (Amit y Schoemaker, 1993). Además, dado su carácter dinámico, no pueden ser consideradas con independencia de su uso (Penrose, 1962). Mientras que los recursos son transferibles entre las empresas, las capacidades son acumulables en el tiempo y, por lo tanto, no son transferibles (Grant, 1991).

Dierickx y Cool (1989) describen cómo las capacidades se construyen con el tiempo a través de una serie de políticas y decisiones de gestión consistentes, entrando a los temas del conocimiento y la experiencia por parte del equipo gestor a ser relevantes.

El marco propuesto se fundamenta en tres capacidades empresariales esenciales:

a. Capacidad de gestión.

b. Capacidad de generar, transferir y utilizar conocimiento.

c. Capacidad para establecer procesos de toma de decisión.

\subsubsection{CAPACIDAD DE GESTIÓN}

Existe un interés en la academia para identificar el papel de la capacidad de gestión en el éxito de la empresa (Mintzberg, 1973; Prahalad y Hamel, 1990), siendo reconocida como una fuente importante para la generación de valor en las organizaciones (Barney, 1991; Penrose, 1959; Castanias y Helfat, 1991).

La capacidad de gestión está apoyada en las habilidades del equipo gerencial (Barney, 1991; Mahoney, 1995) y, aunque no existe un consenso en cuanto a cuáles son, ya que, por ejemplo, para Grant (1991) corresponden a 
capacidades como el monitoreo del desempeño de las diferentes unidades de negocio, la asignación de recursos y la formulación de estrategias, para Crittenden y Crittenden (2008) son las que se desarrollan a partir del ejercicio del liderazgo estratégico, el entendimiento de cuándo y dónde asignar los recursos, el monitoreo vinculado con la recompensa a los logros, y la organización de la estructura estratégica de acuerdo con la cultura corporativa. Se puede observar que algunas de ellas coinciden entre los autores. Johnson y Scholes (2001) hablan de la necesidad de controlar los vínculos en la cadena con el fin de contar con competencias nucleares más sólidas y difíciles de imitar.

Para efectos de la presente investigación, se proponen tres habilidades que debe tener la empresa para el desarrollo de la FYEPI: la formulación e implementación de estrategias, la asignación de recursos y la gestión de proyectos.

Se reconoce una asociación positiva entre la calidad del plan estratégico, la capacidad de ejecución y resultados de la empresa (Hahn y Powers, 2010), "la formulación e implementación de estrategias de gestión exitosas son vistas como esenciales para la generación de capacidades en una empresa" (Sahin, 2001), y sin importar el tamaño de la empresa, estas "necesitan planear de manera estratégica como primer paso hacia el éxito empresarial, ya que obliga al directivo a analizar el contexto real al que se enfrenta la organización" (Herter, 1995; Lopez, 2005, p. 141).

Las PYMES, en un número cada vez mayor, se preocupan más por formular e implementar estrategias debido a la creencia de que esto se va a ver reflejado en mejores desempeños empresariales (Baker, Addams y Davis, 1993; Berry, 1998), aunque este vínculo no sea tan fuerte (French, Kelly y Harrison, 2004).

Maritan (2001) sostiene que el desarrollo e implementación de los métodos para la asignación de recursos son una capacidad importante para la empresa, mientras Segelod
(1998) señala que las buenas prácticas de inversión ayudan a la planificación estratégica y financiera, así como a crear un lenguaje común de la empresa.

La asignación de recursos en los proyectos de inversión puede ser vista como un proceso evolutivo (Noda y Bower, 1996), en donde las empresas tienen rutinas que guían las oportunidades de inversión identificadas y cómo se toman las decisiones de inversión, sirviendo como medio de selección interna (Maritan y Coen, 2004). Las rutinas son "el soporte de las competencias individuales, de la coordinación y del desempeño organizacional. Dado que en parte las rutinas están asociadas al conocimiento tácito, éstas se resisten a toda formalización y difícilmente pueden transferirse" (Jiménez, 2003, pp. 65-66).

Los proyectos requieren de una coordinación e integración, y estos elementos se los proporciona la gestión de proyectos. La gestión de proyectos es "la planificación, seguimiento y control de todos los aspectos de un proyecto y la motivación de todos los que participan en ella para alcanzar los objetivos del proyecto a tiempo y se especifica el costo, la calidad y el rendimiento" (BS6079, 2010). Y, de acuerdo con la definición del Project Management Institute, consiste en "la aplicación de conocimientos, habilidades, herramientas y técnicas a las actividades del proyecto para cumplir con los requisitos del proyecto" (PMI, 2013).

La gestión de proyectos ha evolucionado y madurado en los últimos años; no obstante, su contribución siempre ha sido cuestionada debido a la falta de evidencia empírica, resultando difícil de definir y de medir en la realidad (Thomas y Mullaly, 2007). Pese a lo anterior, para las empresas se puede considerar como un activo estratégico (Jugdev, Mathur y Fung, 2007).

Como mencionan Jugdev et al. (2007, pp. 561), "una inversión en metodologías de gestión de proyectos ayuda a las empresas a alcanzar un cierto grado de éxito del proyecto", y agregan: 
"Las metodologías proporcionan directrices y listas de verificación para asegurar que las prácticas se están siguiendo correctamente y que se alcancen los resultados correctos".

De acuerdo con el PMI (2013), una de las habilidades que debe tener el gerente de proyectos corresponde a la motivación del personal que compone el equipo de proyectos. Así, "un gerente de proyecto puede tener las habilidades necesarias para guiar a un equipo de proyecto a través de las distintas etapas del proyecto $y$ los ciclos de vida del proyecto, pero la motivación del equipo, algo mucho menos tangible, es un elemento esencial de un proyecto exitoso" (Schmid y Adams, 2008, p. 60).

El equipo gerencial, en cabeza del gerente del proyecto, debe estar en capacidad de "comprender lo que motiva a los miembros del equipo y crear un ambiente de respaldo en que las personas trabajen como parte de un equipo de alto desempeño y se sientan estimuladas a sobresalir" (Gido y Clements, 1999, p. 87).

Como primera hipótesis, se tiene:

H1: La capacidad de gestión tiene un efecto positivo en el desempeño de los proyectos.

\subsubsection{CAPACIDAD DE GENERAR, TRANSFERIR Y UTILIZAR CONOCIMIENTO3}

La economía de la información, que "estudia el comportamiento de los agentes cuando se enfrentan al problema de adquisición de información" (Cahuc, 2001, pág. 10), reconoce la dificultad generada por la asimetría y desigualdad en la distribución de la información, dando como resultado la no disponibilidad de esta en el mercado para los agentes que toman decisiones ${ }^{4}$, siendo por lo tanto considerada como un bien escaso y costoso de adquirir (Cahuc, 2001).

Para Davenport y Prusak (2001), las empresas presentan factores de ineficiencia en el mercado del conocimiento, siendo estos la ausencia o información incompleta, la asimetría o desbalance en la tenencia de información entre los diferentes departamentos o áreas de las empresas, y el carácter local de la información, proponiendo que para que las empresas puedan enfrentar estos factores, deben aprender a generar, transferir y utilizar su conocimiento.

Las necesidades de información surgen de "lagunas" en el conocimiento, siendo consideradas como un "obstáculo para que la organización resuelva un problema o aproveche una oportunidad" (Choo, 1999, p. 166).

Para Nonaka, el conocimiento se establece a través de un proceso de creación en espiral, en donde, a partir del conocimiento tácito $^{5}$, aquel "difícil de formalizar y comunicar a otros, se llega al conocimiento explícito, aquel que es formal y sistemático (Nonaka, 1998). Dice Takeuchi (2001) que "el conocimiento explicito puede ser fácilmente procesado por un computador, transmitido electrónicamente o almacenado en bases de datos".

Para disminuir la incertidumbre en la toma de decisión, es necesario tener información, pero para obtenerla se debe buscar y usar (Choo, 1999). La búsqueda "comienza al explorar y compartir ampliamente la información, conectándose con muchas fuentes dentro y fuera

3 Mientras que la información son datos significativos, el conocimiento organizacional es "una mezcla fluida de experiencia estructurada, valores, información contextual e internacionalización experta que proporciona un marco para la evaluación e incorporación de nuevas experiencias e información" (Davenport y Prusask, 2001, p. 6).

4 Para la microeconomía tradicional, una de las características básicas del mercado de competencia perfecta es que los agentes están perfectamente informados.

5 Como menciona Polanyi, "conocemos más de lo que podemos decir" (Nonaka, 1998, p. 27). 
de la organización" (Choo, 1999, p. 171). La exploración tiene simultáneamente un doble movimiento, amplio y estrecho; amplio a la hora de salir a buscar la información en diferentes fuentes, y estrecho cuando, una vez encuentra la fuente, se enfoca para obtener la información. En esta búsqueda, los dos problemas son: a) no poder originar y transferir información desde el exterior a la organización y b) no poder convertir el conocimiento tácito de los expertos en explícito (Choo, 1999).

La generación de conocimiento se puede realizar por diferentes caminos, siendo los más comunes los siguientes (Davenport y Prusask, 2001):

a. por adquisición directa en el mercado;

b. por asignación de recursos como la creación de departamentos de I\&D;

c. la fusión del pensamiento de miembros de diferentes áreas de la empresa en equipos con tareas específicas;

d. la necesidad de adaptación ante el reto de nuevas realidades, $y$

e. las redes establecidas por miembros expertos de la organización, con intereses comunes que se comunican entre sí con frecuencia.

Como estrategia en la consecución de información, las empresas hoy en día presentan cifras considerables en inversiones de tecnologías de información, hardware, software y equipos de telecomunicación (Laudon y Laudon, 2014), y consiguen información a través de firmas de consultoría, ya que estas "pueden disponer de esta información en sus archivos o saber dónde y cómo encontrarla" (Kubr, 2002, p. 15).

Para Pineda et al. (1998), los gerentes de las PYMEs, aunque "utilizan una variedad de fuentes de información, son propensos a depender en gran medida de su propia experiencia y de información de fácil acceso", tendiendo a confiar en fuentes menos formales, tales como la familia y amigos (Cooper, Folta y Woo, 1995), y en las conexiones personales como una fuente de información externa (Johannessen y Dolva, 1995).
Una decisión estratégica para la empresa la constituye el hecho de balancear las fuentes de información internas y externas de acuerdo con sus necesidades y recursos disponibles. Es importante tener en cuenta que tanto las unas como las otras son mutuamente interdependientes y tienen procesos complementarios (Bierly y Chakrabarti, 1996).

El conocimiento siempre se transfiere de manera formal o informal. Sin embargo, "el conocimiento tácito y ambiguo es especialmente difícil de transferir desde la fuente que lo crea hasta las restantes partes de la organización" (Davenport y Prusask, 2001, p. 103), por lo que es importante el trabajo y la comunicación entre los miembros de los equipos multidisciplinarios.

Para Davenport y Prusak (2001), la transferencia de conocimiento en las organizaciones se da a través de la contratación de personas inteligentes y cuando, además, se les permite a estas conversar entre sí, pero que es fácil de decir, es difícil de aplicar y, como ellos anotan, "a menudo, las organizaciones contratan personas brillantes y luego las aíslan o las cargan de tareas que no les permiten dejar un margen de tiempo para conversar $\mathrm{y}$, a veces, ni siquiera para pensar" (Davenport y Prusask, 2001, p. 101).

Para Choo (1999), el uso de la información lleva a la empresa a crear conocimiento que le permite evaluar los beneficios de la misma, mejorando su posicionamiento competitivo; y al tener un mayor conocimiento del comportamiento de la competencia y de sus capacidades internas da como resultado "productos físicos o capacidades reales" (Choo, 1999, p. 177).

Sin embargo, para I-Chin et al. (2013), las PYMES presentan características y prácticas en la gestión de su conocimiento que las lleva a tener problemas a la hora de actuar. Estas se refieren a la falta de recursos, tanto de infraestructura, como humanos. Por lo general, la estructura organizacional gira alrededor del propietario gerente, lo que la hace muy focali- 
zada en él; sus estructuras de gestión son informales y tienen políticas y procesos informales; $y$, de igual manera, su cultura es muy informal.

En la práctica empresarial, la consecución completa de datos es un hecho imposible y, por lo tanto, la estructuración de la información, por lo que el futuro se caracteriza por ser incierto, y al ser incierto genera desequilibrios que los empresarios aprovechan, obteniendo como resultado final utilidades. La realidad es que los empresarios se enfrentan a situaciones que son en extremo únicas; estas situaciones únicas se llaman incertidumbre y son imposibles de medir estadísticamente (Knight, Riesgo, incertidumbre y beneficio, 1947).

El uso de la información busca reducir o eliminar la incertidumbre; no obstante, siempre se va a presentar una diferencia entre la información procesada y la información necesaria para completar una tarea (Frishmmar, 2003). La incertidumbre es una incapacidad para predecir con precisión lo que los resultados de una decisión pueden ser (Duncan, 1972).

Para Barney, existen dos posibilidades para que las fuentes de información se conviertan en una ventaja para la empresa: el análisis del entorno competitivo de la empresa y el análisis de habilidades y capacidades organizacionales controladas por la empresa; y menciona al respecto que "las empresas que tienen éxito en hacer esto, con el tiempo, esperan obtener una mayor rentabilidad en la implementación de estrategias, que las empresas menos informadas, aunque, como siempre, las empresas pueden tener suerte" (Barney, 1986, p. 1.238).

Como segunda hipótesis, se tiene:

H2: La capacidad de generar, transferir y utilizar conocimiento por parte de la empresa PYME tiene un efecto positivo en el desempeño de los proyectos.

\subsubsection{CAPACIDAD PARA ESTABLECER PROCESOS DE TOMA DE DECISIÓN}

Hasta la introducción del tema por Chester Barnard (1937) en la década de los años treinta, la toma de decisiones por parte del gerente $y$ la empresa no era considerada como relevante desde el punto de vista teórico. Simplemente, los negocios se daban. Así, las decisiones eran vistas como simple rutina y le correspondían al personal de nivel bajo de la planta, muy acorde al mundo dibujado por Taylor, en el que se consideraba un tema académico irrelevante (Andreu y Rossanas, 2008).

Ahora, la toma de decisiones no se da como algo que llega de pronto; por el contrario, obedece a un proceso en donde hay entradas y salidas y el principal resultado es la toma de decisión. Según Drucker, es "el resultado de un proceso sistemático, compuesto de elementos claramente definidos y escalonados en una secuencias de etapas bien determinadas" (Drucker, 2004, p. 1).

El proceso de toma de decisión de Mintzberg, Raisinghani y Théorêt (1976) se define como una serie de fases centrales; a) identificación, b) desarrollo y c) selección ${ }^{6}$.

La fase de identificación está compuesta por dos rutinas: reconocimiento de la decisión, en la que las oportunidades, los problemas y las crisis son reconocidos $y$, de otro lado, el diagnóstico, donde la empresa trata de comprender los estímulos que determinan relaciones causaefecto para la situación de decisión.

La fase de desarrollo es en la que más recursos consume la toma de decisiones. Puede ser descrita en términos de dos rutinas básicas: búsqueda y diseño. Con la búsqueda, se trata de encontrar soluciones ya hechas; el diseño se usa para desarrollar soluciones a la medida o para modificar las ya hechas.

6 Este modelo fue desarrollado por Mintzberg, Raisinghani y Théorêt a partir del modelo de toma de decisiones elaborado por Simon (1965). 
Por último, la fase de selección se describe en términos de filtro, evaluación-escogencia y autorización; es un proceso iterativo y de muchas etapas, que requiere evaluaciones profundas de alternativas. Igualmente, Mintzberg et al. (1976) definen tres rutinas adicionales que soportan las tres fases centrales: rutinas de control de decisión, de comunicación de la decisión y de políticas internas.

Sin embargo, y a pesar de ser un proceso inmensamente complejo y dinámico, Pinches (1982) critica la actitud de la academia y los empresarios en cuanto a hacer un mayor énfasis en el punto de la selección, en aspectos como los criterios de decisión, que en los aspectos del proceso de la asignación de recursos, considerando esto como una miopía empresarial.

Según Simon (1972), las empresas presentan una racionalidad limitada, ya que dentro de ellas las personas son las que toman las decisiones. El "hombre económico"7 es el instrumento de la teoría económica, quien toma decisiones seleccionando alternativas óptimas, en ambientes muy específicos y claramente definidos, contrario al "hombre administrativo", quien es una persona con sentimientos, que toma decisiones bajo condiciones de incertidumbre plena, en donde no se pueden analizar todas las alternativas posibles y menos sus probabilidades de ocurrencia (March y Simon, 1994).

Para Simon (1972), el hombre administrativo tiene dos características. En primer lugar, nunca optimiza las soluciones; simplemente, elige sin examinar todas las alternativas posibles, ni sus consecuencias, ya que no le interesa definirlas. Para establecer todas las alternativas, es necesario ejecutar tres pasos: a) la enumeración de todas las estrategias alternativas, b) la determinación de todas las consecuencias que se siguen de cada una de ellas y 3 ) la valoración comparativa de estas series de consecuencias. En el supuesto caso de llegar a conocer todas las posibilidades, "operaria aquí una especie de casualidad inversa: las consecuencias futuras serían las determinantes del comportamiento presente" (Simon, 1972, p. 66), generando una situación completamente contraria a lo que hace, que es formar expectativas de las consecuencias futuras de sus acciones.

En segundo lugar, la toma de decisiones sigue reglas muy "sencillas y manejables" (Simon, 1972, pág. XXIV), que son realizadas por una o varias personas. Las organizaciones están compuestas por personas que, a su vez, se integran en grupos y coaliciones (M intzberg, 1992), convirtiendo la toma de decisiones en una actividad grupal definida a través de procesos administrativos, los cuales en últimas son procesos decisorios. Cuando la toma de decisión se ha realizado muchas veces, se convierte en una rutina.

Concluyen March y Simon que "la mayor parte de las decisiones humanas, ya sean individuales o de organización, se refieren al descubrimiento y selección de alternativas satisfactorias; sólo en casos excepcionales se ocupan del descubrimiento y selección de alternativas óptimas" (March y Simon, 1994, p. 155). Estas decisiones satisfactorias es lo que se conoce como racionalidad limitada o acotada.

El otro hecho fundamental en la toma de decisiones se refiere a que las empresas no tienen un objetivo único, ya que los participantes tienen diferentes intereses que no necesariamente coinciden, presentándose lo que se conoce en la teoría del comportamiento de la empresa

7 El hombre económico se refiere al individuo que maximiza, es decir, "elige la mejor alternativa de entre todas las que tiene a su alcance", y trata con el "mundo real en toda su complejidad" (Simon, 1972, p.xxIV). Tiene un comportamiento maximizador, con conocimiento total y tomando decisiones sobre la base de la maximización (Douma y Schreuder, 2004), en donde su análisis es estático, no dinámico (Dofter, 2006). 
como una coalición de participantes (Douma y Schreuder, 2004). No todos los interesados tienen los mismos objetivos; algunos los definen de manera cuantitativa, y otros, por el contrario, de manera cualitativa. Adler (2000) propone un modelo de decisión de múltiples atributos, en donde las preferencias de las personas que toman las decisiones puedan ser evaluadas, correspondientes a medidas financieras, como recuperación de la inversión y valor presente neto, y medidas no financieras, como la imagen de la empresa, la reducción de la complejidad y la mejora en la información.

El rendimiento financiero se refiere a la capacidad de una empresa para generar entradas netas de efectivo a través de sus operaciones (IFRS, 2013). La toma de decisión para la escogencia de un proyecto conlleva a un análisis de los flujos de caja futuros de la alternativa, esperando que estos sean superiores a los que entregaría para el mismo período el costo de oportunidad. "El objetivo de una decisión de inversión o presupuesto de capital es encontrar activos reales que valgan menos de lo que cuestan" (Brealy, Myers, y Allen, 2006, p. 17).

Lo anterior genera un conflicto que se resuelve a través de una negociación. La negociación es el resultado del hecho propio de las personas que toman decisiones en las empresas, de no tener claro lo que persiguen. Sin embargo, esta negociación tiene precedentes, lo que no constituye una situación que se inicie de "ceros", sino, por el contrario, siempre se revisa lo que la empresa ya ha realizado, dando como resultado un sistema de negociación continua en respuesta a los cambios del entorno (Cyert y March, 1965).

Como tercera hipótesis, se tiene:

H3: La capacidad de establecer un proceso de toma de decisión tiene un efecto positivo en el desempeño de los proyectos.

\subsubsection{DESEMPEÑO DE LOS PROYECTOS}

El desempeño de los proyectos ha sido visto como el cumplimiento con el alcance, el tiempo y los costos, en lo que se ha llamado el "triángulo de hierro" (Belassi y Tukel, 1996); (Atkinson, 1999). Sin embargo, se ha presentado una clara diferencia entre el éxito de la gestión de proyectos, medida a través del alcance, tiempo y costo, y el buen desempeño en general del proyecto (De Wit, 1988).

Según Muller y Turner (2007), dos son los componentes de éxito del proyecto:

a. Los factores de éxito del proyecto, que corresponden a los elementos que influyen en el aumento de la probabilidad de éxito. Estas son variables independientes.

b. Los criterios de éxito del proyecto, que corresponden a las medidas con las cuales se mide dicho éxito. Estas son variables dependientes.

En los últimos años, autores como WueIIner (1990) y Pinto y Pinto (1991) incluyen medidas como la satisfacción de los clientes y del equipo como parte del buen desempeño de los proyectos. Gemünden, Salomo y Krieger (2005) amplían el abanico definiendo el éxito del proyecto, agregando además del alcance, tiempo y costo, una dimensión interna de éxito, en aspectos como los costos del nuevo producto y una dimensión externa, en aspectos como el rendimiento financiero, el cumplimiento de las cuotas de mercado, el aumento de la imagen, entre otros.

Shenhar et al. (2001) logran romper la desarticulación entre el éxito del proyecto y el del producto uniéndolos en una misma estructura, en un concepto multidimensional. Consideran que para que un proyecto tenga éxito debe ser evaluado a lo largo del tiempo, en cuatro dimensiones: eficiencia del proyecto, impacto en el cliente, éxito directo en el negocio, y la preparación para el futuro. Estas dimensiones tienen mayor o menor importancia según el tiempo y el nivel de incertidumbre tecnológica involucrada en el proyecto. 


\subsubsection{ARTICULACIÓN DE LOS TRES ELEMENTOS}

Se propone un marco en donde se articulan tres capacidades claves para el buen desem- peño de la FYEPI: las capacidades de gestión: de generación, transferencia y utilización del conocimiento, y establecimiento de un proceso de toma de decisión.

Gráfico 3. Articulación entre los elementos y el desempeño del proyecto

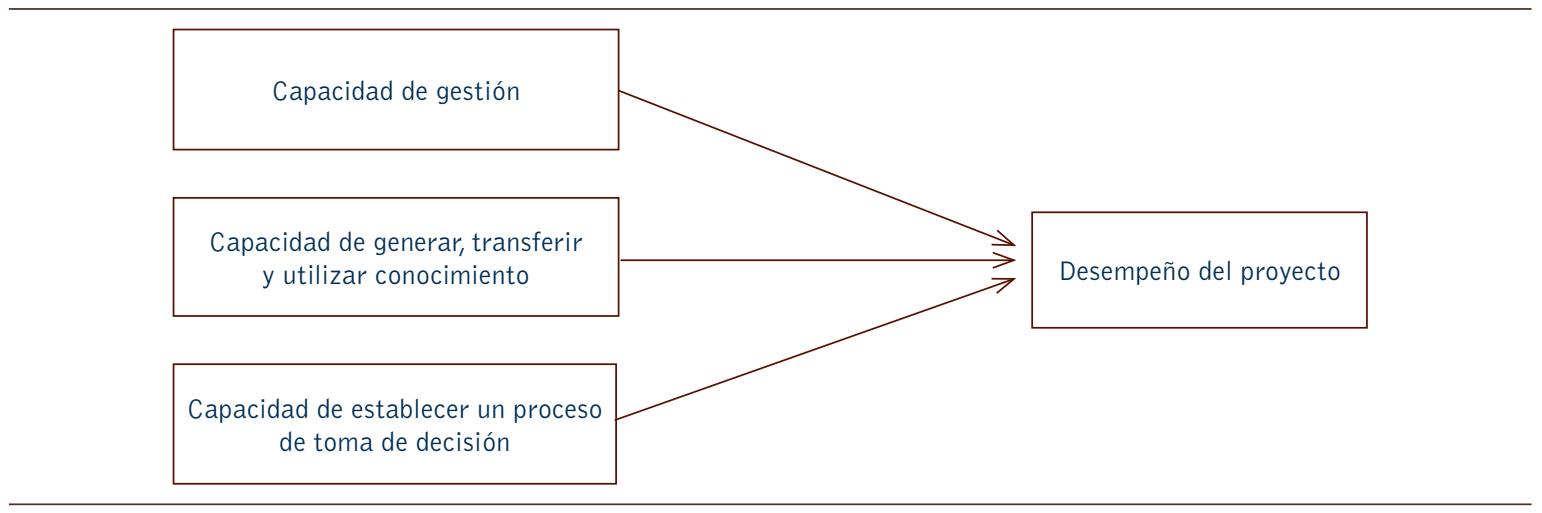

Fuente: Elaboración propia.

\section{METODOLOGÍA}

Para la verificación de las hipótesis y del cumplimiento del objetivo del artículo, se desarrolló una investigación no experimental, de corte transversal, en donde se verifica el marco comprehensivo de manera empírica y causal, utilizando regresión categórica ${ }^{8}$. Esta técnica estadística aplica la regresión "mediante el escalamiento óptimo cuantificando los datos categóricos mediante la asignación de valores numéricos a las categorías y ajustando una ecuación lineal óptima para los valores transformados" (Pérez, 2009, p. 317).

La selección del tratamiento de los datos cualitativos en cuantitativos se obtiene utilizando el escalonamiento óptimo, ya que permite de manera general tratarlos a través de la transformación óptima de escalas cualitativas en valores cuantitativos (De Leeuw, 2006).
Para efectos del desarrollo del formulario, se utiliza la escala ordinal ${ }^{9}$, en donde lo principal es el ordenamiento; se habla de primero, segundo, tercero, en donde no se sabe qué tan cerca o lejos está el primero del segundo puesto. "Los valores de la escala representan categorías o grupos de pertenencia, con cierto orden asociado, pero no una cantidad mensurable. Los números representan una cualidad que se está midiendo, y expresan si una observación tiene más de la cualidad medida que otra" (Orlandoni, 2010).

El formulario fue desarrollado utilizando la escala de Likert de 1 a 7 , en donde 1 es totalmente en desacuerdo; 2, de acuerdo; 3, parcialmente en desacuerdo; 4, neutro; 5, parcialmente de acuerdo, 6, de acuerdo, y 7, totalmente de acuerdo.

Para el contraste de las hipótesis, es necesario contar con datos relativos a las variables 
independientes: capacidad de gestión, capacidad de generar, transferir y utilizar el conocimiento, y capacidad de establecer un proceso de toma de decisión, y la variable dependiente de los resultados de los proyectos de inversión. Igualmente, con el fin de no desvirtuar el análisis de los datos y evitar problemas de interpretación, se ha definido como variable de control el tamaño de las empresas, es decir, las PYMES de acuerdo con lo planteado anteriormente.
El formulario fue encabezado con la siguiente frase: "A lo largo de estos años, la empresa con relación a la formulación y evaluación de sus proyectos de inversión ha tenido la capacidad para: ..........".

A continuación, se presentan los diferentes autores que apoyan cada una de las diferentes variables evaluadas.

Tabla 4. Preguntas relacionadas con la capacidad de gestión

\begin{tabular}{|c|c|c|}
\hline $\mathrm{N}^{\circ}$ & Pregunta & Autor \\
\hline 1 & Formular e implementar estrategias con resultados de calidad. & $\begin{array}{l}\text { Grant (1991), Hahn y } \\
\text { Powers (2010) }\end{array}$ \\
\hline 2 & $\begin{array}{l}\text { Reconocer como esencial la formulación e implementación de estrategias de gestión } \\
\text { exitosas. }\end{array}$ & Sahin (2001) \\
\hline 3 & Asignar recursos a los nuevos proyectos de inversión. & $\begin{array}{l}\text { Grant (1991), Maritan } \\
\text { (2001), Segelod (1998) }\end{array}$ \\
\hline 4 & Generar rutinas para la asignación de recursos a los nuevos proyectos de inversión. & $\begin{array}{l}\text { Noda y Bower (1996) } \\
\text { Maritan y Coen (2004) }\end{array}$ \\
\hline 5 & Gestionar los proyectos que se van a implementar. & BS6079 (2010), PMI (2013) \\
\hline 6 & Planificar, seguir y controlar todos los aspectos del proyecto. & $\begin{array}{l}\text { Grant (1991), BS6079 (2010), } \\
\text { PMI (2013) }\end{array}$ \\
\hline 7 & $\begin{array}{l}\text { Motivar a todos los que participan en el proyecto para alcanzar los objetivos a } \\
\text { tiempo y dentro del costo, la calidad y el rendimiento. }\end{array}$ & Schmid y Adams (2008) \\
\hline
\end{tabular}

Fuente: Elaborada por el autor con base en Grant (1991), Hahn y Powers (2010), Sahin (2001), Noda y Bower (1996), Maritan y Coen (2004), Maritan (2001), Segelod (1998), BS6079 (2010), PMI (2013) y Schmid y Adams (2008).

Tabla 5. Preguntas relacionadas con la capacidad de generar, transferir y utilizar el conocimiento

\begin{tabular}{|c|c|c|}
\hline $\mathrm{N}^{\circ}$ & Pregunta & Autor \\
\hline 1 & Generar conocimiento con información elaborada específicamente para el proyecto. & Choo (1999). \\
\hline 2 & Generar conocimiento por adquisición directa en el mercado & Davenport y Prusak (2001) \\
\hline 3 & Generar conocimiento internamente con base en la información de los empleados. & Choo (1999). \\
\hline 4 & $\begin{array}{l}\text { Generar conocimiento internamente con base en la información de la gerencia y los } \\
\text { accionistas. }\end{array}$ & Pineda et al. (1998) \\
\hline 5 & $\begin{array}{l}\text { Transferir conocimiento entre las diferentes áreas de la empresa involucradas en el } \\
\text { proyecto. }\end{array}$ & $\begin{array}{l}\text { Choo (1999), Davenport y } \\
\text { Prusak (2001) }\end{array}$ \\
\hline 6 & $\begin{array}{l}\text { Utilizar conocimiento generado y trasferido en la empresa para el desarrollo de los } \\
\text { proyectos. }\end{array}$ & Choo (1999). \\
\hline 7 & $\begin{array}{l}\text { Reducir la incertidumbre causada por la falta de información y conocimiento acerca } \\
\text { de los nuevos proyectos. }\end{array}$ & $\begin{array}{l}\text { Frishmmar (2003), Duncan } \\
\text { (1972). }\end{array}$ \\
\hline
\end{tabular}

Fuente: Elaborada por el autor con base en Choo (1999), Davenport y Prusak (2001) y Pineda et al. (1998). 
Tabla 6. Preguntas relacionadas con la capacidad de establecer un proceso de toma de decisión

\begin{tabular}{c|l|l}
\hline$N^{0}$ & \multicolumn{1}{|c}{ Pregunta } & \multicolumn{1}{|c}{ Autor } \\
\hline 1 & Establecer un proceso de toma de decisión. & $\begin{array}{l}\text { Mintzberg, Raisinghani y } \\
\text { Théorêt (1976) }\end{array}$ \\
\hline 2 & Establecer una etapa de identificación dentro del proceso de toma de decisión. & $\begin{array}{l}\text { Mintzberg, Raisinghani y } \\
\text { Théorêt (1976) }\end{array}$ \\
\hline 3 & Establecer una etapa de desarrollo dentro del proceso de toma de decisión. & $\begin{array}{l}\text { Mintzberg, Raisinghani y } \\
\text { Théorêt (1976) }\end{array}$ \\
\hline 4 & Establecer una etapa de selección dentro del proceso de toma de decisión. & $\begin{array}{l}\text { Mintzberg, Raisinghani y } \\
\text { Théorêt (1976) }\end{array}$ \\
\hline 5 & $\begin{array}{l}\text { Definir rutinas para el control del proceso de decisión y la comunicación de los } \\
\text { cambios que allí ocurran. }\end{array}$ & $\begin{array}{l}\text { Mintzberg, Raisinghani y } \\
\text { Théorêt (1976) }\end{array}$ \\
\hline 6 & Tomar decisiones analizando pocas alternativas. & $\begin{array}{l}\text { Simon (1972), March y } \\
\text { Simon (1994). }\end{array}$ \\
\hline 7 & Definir criterios de evaluación financiera. & Adler (2000) \\
\hline 8 & Definir criterios de evaluación no financieros. & Adler (2000) \\
\hline 9 & Definir criterios únicos de evaluación entre los interesados. & Douma y Scheuder (2004). \\
\hline
\end{tabular}

Fuente: Elaborada por el autor con base en Mintzberg, Raisinghani y Théorêt (1976), Simon (1972), March y Simon (1994), Adler ((200) y Douma y Sheuder (2004).

Para el desempeño de los proyectos, se inicia la pregunta con la siguiente frase: "En los últimos tres años, y en comparación con la competencia, el desempeño de los proyectos de la empresa han sido favorables en términos de".
Los resultados obtenidos para las seis preguntas que se establecen en la tabla 7 fueron promediados en un solo indicador, esto con el fin de tener una sola variable dependiente dentro de la regresión.

Tabla 7. Preguntas relacionadas con los resultados del proyecto

\begin{tabular}{l|l|l}
\hline N. ${ }^{\circ}$ & \multicolumn{1}{|c}{ Pregunta } & \multicolumn{1}{c}{ Autor } \\
\hline 1 & Lograr el alcance. & Belassi y Tukel (1996) Atkinson (1999) \\
\hline 2 & Obtener eficiencia en los costos de ejecución. & Belassi y Tukel (1996) Atkinson (1999) \\
\hline 3 & Obtener eficiencia en los tiempos de ejecución. & Belassi y Tukel (1996) Atkinson (1999) \\
\hline 4 & Lograr los impactos percibidos por nuestros clientes. & Shenhar et al. (2001) \\
\hline 5 & Lograr los resultados directos en la empresa. & Shenhar et al. (2001) \\
\hline 6 & Preparar para el futuro a la empresa. & Shenhar et al. (2001) \\
\hline
\end{tabular}

Fuente: Elaborada por el autor con base en Belassi y Tukel (1996), Atkinson (1999) y Shenhar et al. (2001).

Las encuestas fueron aplicadas a empresas PYMES del sector de servicios de la ciudad de Bogotá, entre los meses de octubre y noviembre de 2014, realizando 206 encuestas vía telefónica, en donde se obtuvo un error del $6,96 \%$ de la muestra y una fiabilidad de la escala calculada con el coeficiente alfa de Cronbach de 0,854, aceptable para este tipo de análisis.

Los sectores económicos encuestados fueron los siguientes: 
Tabla 8. Sectores económicos de las empresas encuestadas

\begin{tabular}{c|l|c|c}
\hline Código & \multicolumn{1}{|c|}{ Actividad económica } & Frecuencia & Porcentaje \\
\hline 1 & $\begin{array}{l}\text { a. Servicios de alojamiento en hoteles, campamentos y otros tipos de hospedaje no } \\
\text { permanente. }\end{array}$ & 14 & 6,80 \\
\hline 2 & b. Expendio de alimentos preparados en el sitio de venta y bebidas alcohólicas. & 28 & 13,59 \\
\hline 3 & c. Actividades complementarias y auxiliares al transporte. & 10 & 4,85 \\
\hline 4 & d. Actividades de agencias de viajes. & 14 & 6,80 \\
\hline 5 & e. Actividades postales y de correo. & 12 & 5,83 \\
\hline 6 & f. Telecomunicaciones. & 2 & 0,97 \\
\hline 7 & g. Actividades inmobiliarias, alquiler de maquinaria y eqpo sin operario. & 38 & 18,45 \\
\hline 8 & h. Informática y actividades conexas. & 23 & 11,17 \\
\hline 9 & i. Obtención y suministro de personal, seguridad y aseo. & 10 & 4,85 \\
\hline 10 & j. Otras actividades empresariales. & 19 & 9,22 \\
\hline 11 & k. Servicios de educación superior privada. & 6 & 2,91 \\
\hline 12 & l. Actividades relacionadas con la salud humana privada. & 18 & 8,74 \\
\hline 13 & m. Actividades de radio, televisión y de agencias de noticias. & 12 & 5,83 \\
\hline & & 206 & 100,00 \\
\hline
\end{tabular}

Fuente: Elaboración propia.

\section{RESULTADOS}

El $76,70 \%$ de las empresas encuestadas, es decir 158 , son pequeñas y el $23,30 \%$ son medianas, y solo el $1 \%$, es decir, dos empresas, no ha realizado ningún proyecto de inversión durante los últimos tres años. Así mismo, el $6,80 \%$ de las empresas no documenta de manera escrita sus proyectos.

Ante la pregunta acerca de las razones para realizar sus proyectos, las empresas consultadas expresaron estar de "acuerdo" y "totalmente de acuerdo", en un $65 \%$, en cuanto a que los proyectos de inversión desarrollados últimamente buscan mejorar la eficiencia en los costos; en un 63,1\% buscan establecer reemplazos de la maquinaria y equipos existentes; en un
$47,60 \%$ buscan externalizar o internalizar actividades o procesos; en un $48,50 \%$, introducir al mercado nuevos productos, y en un $51,50 \%$ buscan conquistar nuevos mercados. Como se puede observar, las dos primeras alternativas, temas de eficiencia y reemplazo de maquinaria y equipos, son las más frecuentes.

Con relación a la primera hipótesis planteada, H1: La capacidad de gestión tiene un efecto positivo en el desempeño de los proyectos, los resultados del análisis de la varianza son presentados en la tabla 9. Estos muestran que la varianza del modelo $(4,781)$ es mayor a la varianza del error $(, 749)$, lo que indica que los diferentes predictores separada y conjuntamente predicen el éxito de los proyectos. 
Tabla 9. Anova: Capacidad de gestión

\begin{tabular}{l|c|c|c|c|c}
\hline & Suma de cuadrados & Grados de libertad & Media cuadrática & Prueba F & Significancia estadística \\
\hline Regresión & 62,158 & 13 & 4,781 & 6,382 &, 000 \\
\hline residual & 143,842 & 192 &, 749 & & \\
\hline Total & 206,000 & 205 & & & \\
\hline
\end{tabular}

Utilizando el análisis de regresión categórica, en la tabla 10 se presentan los resultados de las variables que conforman la capacidad de gestión. Se establece que tres variables: 1 ) la capacidad de gestión para asignar recursos a los nuevos proyectos de inversión; 2) la capacidad de gestión para gestionar los proyectos a implementar, y 3) la capacidad de gestión para planificar, seguir y controlar todos los aspectos del proyecto, son fuertes predictores del éxito de los proyectos, ya que presentan valores de significancia menores a 0,05. Por el contrario, el resto de variables presentan valores superiores a 0,05 , es decir, no son predictores significativos del éxito de los proyectos. Se resalta que a diferencia del resto de las variables, la de capacidad de gestión para motivar a todos los que participan en el proyecto para alcanzar los objetivos a tiempo y dentro del costo, la calidad y el rendimiento tiene una incidencia negativa en los resultados, no obstante es poco significativa.

Tabla 10. Coeficientes de la regresión categórica que indican la significancia de las variables de la capacidad de gestión en los resultados de los proyectos

\begin{tabular}{|c|c|c|c|c|c|}
\hline \multicolumn{6}{|c|}{ Coeficientes } \\
\hline & \multicolumn{2}{|c|}{ Coeficientes tipificados } & \multirow[b]{2}{*}{$\begin{array}{l}\text { Grados de } \\
\text { libertad }\end{array}$} & \multirow[b]{2}{*}{ Prueba F } & \multirow[b]{2}{*}{$\begin{array}{l}\text { Significancia } \\
\text { estadística }\end{array}$} \\
\hline & Beta & $\begin{array}{l}\text { Bootstrap } \\
(1000) \\
\text { Estimación } \\
\text { de error } \\
\text { típico }\end{array}$ & & & \\
\hline $\begin{array}{l}\text { Formular e implementar estrategias con resultados de } \\
\text { calidad. }\end{array}$ & 0,083 & 0,145 & 2 & 0,330 & 0,719 \\
\hline $\begin{array}{l}\text { Reconocer como esencial la formulación e implementación } \\
\text { de estrategias de gestión exitosas. }\end{array}$ & 0,107 & 0,103 & 3 & 1,064 & 0,366 \\
\hline Asignar recursos a los nuevos proyectos de inversión. & 0,256 & 0,087 & 2 & 8,580 & 0,000 \\
\hline $\begin{array}{l}\text { Generar rutinas para la asignación de recursos a los nuevos } \\
\text { proyectos de inversión. }\end{array}$ & 0,083 & 0,123 & 2 & 0,461 & 0,632 \\
\hline Gestionar los proyectos que se van a implementar. & 0,173 & 0,081 & 1 & 4,520 & 0,035 \\
\hline $\begin{array}{l}\text { Planificar, seguir y controlar todos los aspectos del } \\
\text { proyecto. }\end{array}$ & 0,237 & 0,115 & 2 & 4,203 & 0,016 \\
\hline $\begin{array}{l}\text { Motivar a todos los que participan en el proyecto para } \\
\text { alcanzar los objetivos a tiempo y dentro del costo, la calidad } \\
\text { y el rendimiento. }\end{array}$ & $-0,116$ & 0,173 & 1 & 0,449 & 0,504 \\
\hline
\end{tabular}


Con respecto a la segunda hipótesis planteada, H2: La capacidad de generar, transferir y utilizar conocimiento por parte de la empresa PYME tiene un efecto positivo en el desempeño de los proyectos. Los resultados del análisis de la varianza son presentados en la tabla 11 . Estos muestran que la varianza del modelo $(7,253)$ es considerablemente mayor a la varianza del error $(, 616)$, lo que indica que los diferentes predictores separada y conjuntamente predicen bastante bien el éxito de los proyectos.

Utilizando el análisis de regresión categórica, en la tabla 12 se presentan los resultados de las variables que conforman la capacidad de generar, transferir, y utilizar conocimiento. Se establece que tres variables: 1 ) generar conocimiento con información elaborada especí- ficamente para el proyecto; 2) generar conocimiento internamente con base en la información de la gerencia y los accionistas, y 3 ) transferir conocimiento entre las diferentes áreas de la empresa involucradas en el proyecto, son fuertes predictores del éxito de los proyectos, ya que presentan valores de significancia bastante menores a 0,05. Por el contrario, el resto de variables presentan valores superiores a 0,05 , es decir, no son predictores significativos del éxito de los proyectos.

Se resalta que las variables correspondientes a generar conocimiento por adquisición directa en el mercado y utilizar conocimiento generado y trasferido en la empresa para el desarrollo de los proyectos tienen una incidencia negativa en los resultados de los proyectos.

Tabla 11. Anova: Capacidad de generar, transferir y utilizar conocimiento

\begin{tabular}{l|c|c|c|c|c}
\hline & $\begin{array}{c}\text { Suma de } \\
\text { cuadrados }\end{array}$ & Grados de libertad & Media cuadrática & Prueba F & $\begin{array}{c}\text { Significancia } \\
\text { estadística }\end{array}$ \\
\hline Regresión & 87,031 & 12 & 7,253 & 11,766 & 0,000 \\
\hline Residual & 118,969 & 193 &, 616 & & \\
\hline Total & 206,000 & 205 & & & \\
\hline
\end{tabular}

Tabla 12. Coeficientes de la regresión categórica que indican la significancia de las variables de la capacidad de generar, transferir y utilizar conocimiento en los resultados de los proyectos

\begin{tabular}{|c|c|c|c|c|c|}
\hline \multicolumn{6}{|c|}{ Coeficientes } \\
\hline & \multicolumn{2}{|c|}{ Coeficientes tipificados } & \multirow[b]{2}{*}{$\begin{array}{l}\text { Grados de } \\
\text { libertad }\end{array}$} & \multirow[b]{2}{*}{$\begin{array}{l}\text { Prueba } \\
\text { F }\end{array}$} & \multirow[b]{2}{*}{$\begin{array}{l}\text { Significancia } \\
\text { estadística }\end{array}$} \\
\hline & Beta & $\begin{array}{l}\text { Bootstrap } \\
\text { (1000) } \\
\text { Estimación de } \\
\text { error típico }\end{array}$ & & & \\
\hline $\begin{array}{l}\text { Generar conocimiento con información elaborada } \\
\text { específicamente para el proyecto. }\end{array}$ & 0,162 & 0,063 & 2 & 6,68 & 0,00 \\
\hline Generar conocimiento por adquisición directa en el mercado. & $-0,287$ & 0,196 & 2 & 2,13 & 0,12 \\
\hline $\begin{array}{l}\text { Generar conocimiento internamente con base en la } \\
\text { información de los empleados. }\end{array}$ & 0,06 & 0,098 & 1 & 0,37 & 0,54 \\
\hline $\begin{array}{l}\text { Generar conocimiento internamente con base en la } \\
\text { información de la gerencia y los accionistas. }\end{array}$ & 0,738 & 0,15 & 2 & 24,05 & 0,00 \\
\hline $\begin{array}{l}\text { Transferir conocimiento entre las diferentes áreas de la } \\
\text { empresa involucradas en el proyecto. }\end{array}$ & 0,287 & 0,087 & 2 & 10,77 & 0,00 \\
\hline $\begin{array}{l}\text { Utilizar conocimiento generado y trasferido en la empresa } \\
\text { para el desarrollo de los proyectos. }\end{array}$ & $-0,211$ & 0,167 & 2 & 1,60 & 0,20 \\
\hline $\begin{array}{l}\text { Reducir la incertidumbre causada por la falta de } \\
\text { información y conocimiento acerca de los nuevos proyectos. }\end{array}$ & 0,132 & 0,11 & 1 & 1,42 & 0,23 \\
\hline
\end{tabular}


Con respecto a la tercera hipótesis planteada, H3: La capacidad de establecer un proceso de toma de decisión tiene un efecto positivo en el desempeño de los proyectos, los resultados del análisis de la varianza son presentados en la tabla 13. Estos muestran que la varianza del modelo $(3,838)$ es mayor a la varianza del error (,681), lo que indica que los diferentes predictores separada y conjuntamente predicen el éxito de los proyectos.

Utilizando el análisis de regresión categórica, en la tabla 14 se presentan los resultados de las variables que conforman la capacidad de establecer un proceso de toma de decisión. Se establece que dos variables: 1) determinar un proceso de toma de decisión y 2) definir criterios de evaluación financiera son fuertes predictores del éxito de los proyectos, ya que presentan valores de significancia menores a 0,05 . Por el contrario, el resto de las variables presentan valores superiores a 0,05, es decir, no son predictores significativos del éxito de los proyectos.

Se resalta que las variables correspondientes a establecer una etapa de desarrollo dentro del proceso de toma de decisión y tomar decisiones analizando pocas alternativas tienen una incidencia negativa en los resultados de los proyectos; sin embargo, son predictores poco significativos.

Tabla 13. Anova: Capacidad de establecer un proceso de toma de decisión

\begin{tabular}{l|c|c|c|c|c}
\hline & $\begin{array}{c}\text { Suma de } \\
\text { cuadrados }\end{array}$ & Grados de libertad & Media cuadrática & Prueba F & $\begin{array}{c}\text { Significancia } \\
\text { estadística }\end{array}$ \\
\hline Regresión & 80,607 & 21 & 3,838 & 5,632 &, 000 \\
\hline residual & 125,393 & 184 &, 681 & & \\
\hline Total & 206,000 & 205 & & & \\
\hline
\end{tabular}

Tabla 14. Coeficientes de la regresión categórica que indican la significancia de las variables de la capacidad de establecer un proceso de toma de decisión en los resultados de los proyectos

\begin{tabular}{|c|c|c|c|c|c|}
\hline \multicolumn{6}{|c|}{ Coeficientes } \\
\hline & \multicolumn{2}{|c|}{ Coeficientes tipificados } & \multirow[b]{2}{*}{$\begin{array}{l}\text { Grados de } \\
\text { libertad }\end{array}$} & \multirow[b]{2}{*}{$\begin{array}{l}\text { Prueba } \\
\text { F }\end{array}$} & \multirow[b]{2}{*}{$\begin{array}{l}\text { Significancia } \\
\text { estadística }\end{array}$} \\
\hline & Beta & $\begin{array}{l}\text { Bootstrap } \\
(1000) \\
\text { Estimación de } \\
\text { error típico }\end{array}$ & & & \\
\hline Establecer un proceso de toma de decisión. & 0,281 & 0,149 & 2 & 3,575 & 0,030 \\
\hline $\begin{array}{l}\text { Establecer una etapa de identificación dentro del proceso de } \\
\text { toma de decisión. }\end{array}$ & 0,134 & 0,127 & 3 & 1,118 & 0,343 \\
\hline $\begin{array}{l}\text { Establecer una etapa de desarrollo dentro del proceso de } \\
\text { toma de decisión. }\end{array}$ & $-0,027$ & 0,14 & 1 & 0,036 & 0,849 \\
\hline $\begin{array}{l}\text { Establecer una etapa de selección dentro del proceso de } \\
\text { toma de decisión. }\end{array}$ & 0,102 & 0,187 & 2 & 0,297 & 0,743 \\
\hline $\begin{array}{l}\text { Definir rutinas para el control del proceso de decisión y la } \\
\text { comunicación de los cambios que allí ocurran. }\end{array}$ & 0,125 & 0,106 & 3 & 1,402 & 0,244 \\
\hline Tomar decisiones analizando pocas alternativas. & $-0,122$ & 0,121 & 2 & 1,020 & 0,363 \\
\hline Definir criterios de evaluación financiera. & 0,243 & 0,077 & 4 & 9,930 & 0,000 \\
\hline Definir criterios de evaluación no financieros. & 0,07 & 0,172 & 2 & 0,165 & 0,848 \\
\hline Definir criterios únicos de evaluación entre los interesados. & 0,075 & 0,13 & 2 & 0,337 & 0,714 \\
\hline
\end{tabular}




\section{CONCLUSIONES}

Se presenta una nueva visión de la Formulación y Evaluación de Proyectos de Inversión, proponiendo un marco comprehensivo para las pequeñas y medianas empresas del sector de servicios en la ciudad de Bogotá, que permite a los empresarios entender y obtener mejores desempeños en sus proyectos.

El marco propuesto está basado en la teoría de recursos y capacidades, en donde, para obtener una verdadera ventaja competitiva sostenible en el tiempo, es necesario que los recursos se conviertan en capacidades, y estas en desempeño positivo para la empresa (Mahoney y Padian, 1992).

De acuerdo con los resultados, se ha encontrado que las capacidades de gestión-las de generar, transferir y utilizar conocimiento por parte de la empresa PYME, y el establecer un proceso de toma de decisión- tienen un efecto positivo en el desempeño de los proyectos.

De manera particular, para cada una de estas capacidades, algunas de las variables que las componen son mejores predictores. Para las variables que conforman la capacidad de gestión, se establecen tres: la capacidad de gestión para asignar recursos a los nuevos proyectos de inversión; la capacidad para gestionar los proyectos que se van a implementar, y la capacidad para planificar, seguir y controlar todos los aspectos del proyecto, son fuertes predictores del desempeño de los mismos.

En términos generales, los resultados encontrados en las empresas PYMES de servicios concentran sus esfuerzos más en la asignación de los recursos y la gestión de los proyectos que en la formulación de estrategias; esto último en oposición a lo mencionado por Grant (1991), que articula estas tres variables como ejes de las capacidades de gestión, pero muy de acuerdo con Mintzberg (1994), que argumenta que las organizaciones, al actuar en entornos inciertos, no justifican el desarrollo de estrategias explícitas, ya que bloquean la visión general del entorno y hacen a un lado el espíritu innovador del empresario.

De la misma forma, los resultados están en concordancia con lo expresado por Maritan (2001) y Segelod (1998) en lo referente a la asignación de recursos, y a lo señalado por el British Standard Institute (BS6079, 2010) y el PMI (2013).

Para las variables que conforman la capacidad de generar, transferir y utilizar conocimiento, se establecen tres: generar conocimiento con información elaborada específicamente para el proyecto; generar conocimiento internamente con base en la información de la gerencia y los accionistas, y transferir conocimiento entre las diferentes áreas de la empresa involucradas en el proyecto, que son fuertes predictores del desempeño.

Los resultados están de acuerdo con lo mencionado por Choo (1999), según el cual, para disminuir la incertidumbre en la toma de decisión es necesario tener información, pero para obtenerla se debe buscar y usar; y con lo expresado por Pineda et al. (1998), que establecen que las principales fuente de información son la gerencia y los accionistas; esto muy de acuerdo con lo señalado por Bello (2013). De igual manera, los empresarios encuentran relevante la utilización del conocimiento generado y transferido en la empresa.

Para las variables que conforman la capacidad de establecer un proceso de toma de decisión, se definen el proceso en sí y los criterios de evaluación financiera, que son fuertes predictores del desempeño de los proyectos.

Los empresarios reconocen como relevante la capacidad para establecer un proceso de toma de decisión, en concordancia con lo expresado por Drucker (2004), Mintzberg, Raisinghani y Théorêt (1976), y Simon (1965). Sin embargo, no es claro cuáles elementos del proceso son relevantes; simplemente, mencionan que lo hacen, pero no son concluyentes en cuanto al cómo. 
A diferencia de lo propuesto por Adler (2000), los resultados establecen la utilización solo de criterios de evaluación financiera, en lugar de una combinación de atributos financieros y no financieros, donde las preferencias de las personas que toman las decisiones puedan ser evaluadas.

Para obtener los mejores desempeños en sus proyectos, las pequeñas y medianas empresas del sector de servicios en la ciudad de Bogotá requieren contar para la Formulación y Evaluación de Proyectos de Inversión con las capacidades de gestión; de generación, transferencia y utilización de conocimiento, y el establecimiento de un proceso de toma de decisión.

No obstante, al momento de las conclusiones, se debe recordar que, por ser un estudio local, solo para las empresas PYMES del sector de servicios de la ciudad de Bogotá, con un error del $6,96 \%$ de la muestra, los resultados no pueden ser generalizados.

\section{BIBLIOGRAFÍA}

ACOPI (2006). La PYME y sus aportes al desarrollo de Colombia. Bogotá D.C.: Unión Gráfica Ltda.

Adler, R. W. (2000). "Strategic Investment Decision Techniques: The Old and the New", en Business Horizons, vol. 43, n. ${ }^{\circ}$ 6, pp.15-22.

Amit, R. y Schoemaker, P. (1993). "Strategic assets and organizational rent", en Strategic Management Journal, 14(1):33-46.

Andreu, R. y Rossanas, J. M. (2008). "Toma de Decisiones y Actos Directivos", en IESE Revista de Antiguos Alumnos, 53 - 59.

Ang, J. (1991). "Small Business Uniqueness and the Theory of Financial Management", en The Journal of Small Business Finance, 1 (1) , 1-13.

Araujo, D. (2012). Proyectos de Inversión. México, D. F.: Trillas .

Arzubiaga, U., Iturralde, T. y Maseda, A. (2012). "La Medición de la Orientación Emprendedora en las empresas de familia: una revisión crítica de la literatura", en Revista Empresa Familiar, 2 (2), $57-71$.

Atkinson, R. (1999). "Project management: cost, time and quality, two best guesses and a phenomenon. Its time to accept other success criteria", en International Journal of Project Management, 17, 337-342 (1999).

Baca, G. (1990). Evaluación de Proyectos Análisis y Administración de Riesgo. México D. F.: McGrawHill Interamericana de México, S.A. de CV.

Baca, G. (2006). Formulación y Evaluación de Proyectos Informáticos. México, D. F.: McGraw - Hill.

Baker, W., Addams, H. y Davis, B. (1993). "Business planning in successful small firms", en Long Range Planning, 26 (6), 82-88. .

Baldwin, C. y Clark, K. (1992). "Capabilities and Capital Invesment: New Perspectives on Capital Budgeting", en Journal of Applied Corporate Finance, 5 (2): pp. 67-82.

Baldwin, C. y Clark, K. (1994). "Capitalbudgeting System and Capabilities Invesment in US Compamies after the Second World War", en Business History Review, pp. 79-109.

Banco Mundial (2008). Financiamiento Bancario para la pequeña y medianas empresas (PYMES). Bogotá: Banco Mundial.

Barnard, C. I. (1937). The Functions of the Executive. Boston, Massachusetts: Harvard Business School Press.

Barney, J. B. (1986). "Strategic factor markets: expectations, luck, and business strategy", en Management Science, 32(10): 1231-1241.

Barney, J. B. (1991). "Firm Resources and Sustained Competitive Advantage", en Journal of Management, 17(1):99-121.

Becerra, M. (2009). Theory of the Fiirm for Strategy Management. Cambridge: Cambridge University Press.

Belassi, W. y Tukel, 0. I. (1996). "A new framework for determining critical success/failure factors in projects", en International Journal of Project Management, 14(3), 141-151.

Bello, C.J. (2013). Producción y operaciones aplicadas a las pymes. Bogotá D.C.: ECOE Ediciones. 
Berry, M. (1998). "Strategic planning in small high tech companies", en Long Range Planning, 31 (3), 455-466. .

Bierly, P. y Chakrabarti, A. (1996). "Generic knowledge strategies in the U.S. pharmaceutical industry", en Strategic Management Journal, 17: 123-125. .

Bower, J. L. (1995). "Gestión de Asignación de Recursos", en Oficio y Arte de la Gerencia (págs. 136-147). Bogotá, D. C.: Editorial Norma S. A.

Brealy, F., Myers, S. y Allen, R. A. (2006). Principios de Finanzas Corporativas. Madrid: McGraw-Hill/Inteamericana de España.

Brunet, I. (2011). Creación de Empresas. Emprendimiento e innovación. Madrid - España: Ra-ma Editorial.

BS6079 (2010). Project management. Principles and guidelines for the management of projects. London: British Estándar Institute.

Cahuc, P. (2001). La Nueva Microeconomía. Bogotá: Colombia: Alfaomega S.A. .

Castanias, R. P. y Helfat, C. (1991). "Managerial Resources and Rents", en Journal of Management, 17(11), pp. 155-171.

Chan, I. y Chao, C. K. (2008). "Knowledge management in small and medium-sized enterprises", en Communications of the ACM, 51(4), 83-88. .

Choo, C. W. (1999). La Organización Inteligente. México: Oxford University Press México, S. A. de C. V.

Cooper, A. C., Folta, T. B. y Woo, C. (1995). WEntrepreneutial Information Search", en Journal of Business Venturing, 10, 107-120.

Crawford, L., Hobbs, B.y Turner, J. R. (2005). Project Categorization Systems. Newton Square, PA, USA: PMI.

Crittenden, V. L. y Crittenden, W. (2008). "Building a capable organization: The eight levers of strategy implementation", en Business Horizons, 51(4), 301-309.

Cyert, R. M. y March, J. G. (2001). A Behavioral Theory of the Firm. Melden, Massachusetts: Blackwell Publisher Ltd.

Davenport, T. H. y Prusask, L. (2001). Conocimiento en acción. Buenos Aires: Prentice Hall.
Dávila, J. C. (2009). Capacidades dinámicas: un acercamiento a las teorías contemporáneas de la firma. Evidencias de dos organizaciones que operan en Colombia. Bogotá - Colombia: Monografías de Administración. Facultad de Administración. Universidad de los Andes.

De Leeuw, J. (2006). "Nonlinear principal component analysis and related techniques", en M. Greenacre y J. Blasius, Multiple Correspondence Analysis and Related Techniques (págs. 107-133). Boca Raton, FL: Chapman \& Hall.

De Wit, A. (1988). "Measurement of project success", en International Journal of Project Management, vol. 6.

Dean, J. (1973). Política de Inversiones. Barcelona: Editorial Labor.

Dierickx, I. y Cool, K. (1989). "Asset Stock Accumulation and Sustainability of Competitive Advantage", en Management Science, 35(12): 1504-1511.

Dofter, K. (2006). "The Origins of Meso Economics Schumpeters' Legacy", en Papers of Economics and Evolutions, 0610: 1-43.

Douma, S.y Schreuder, H. (2004). Enfoques económicos para el análisis de las organizaciones. Madrid - España: Civitas Ediciones.

Drucker, P. F. (2004). "La decisión eficaz", en H. B. Review, Toma de Decisiones (págs. 1 - 22). Buenos Aires: Ediciones Deusto.

Duncan, R. (1972). "Characteristics of organizational environments and perceived environmental uncertainty", en Administrative Science Quarterly, vol. 17, n. ${ }^{\circ} 3$, pp.313-27. .

Eisenhardt, K. M. y Zbaracki, M. J. (1992). "Strategic Decision Making", en Strategic Management Journal, vol. 13, 17-37.

Etimologías (s.f.). Origen de las Palabras. Recuperado el 20 de abril de 2014, de http://etimologias.dechile.net/?proyecto

Ferrero, C.y Stumpo, G. (2010). "Las PyMES en el laberinto de las políticas", en C. Ferrero, y G. Stumpo, Políticas de aporyo a las PYMES en América Latina (pp. 17-44). Santiago de Chile: Naciones Unidas. 
French, S., Kelly, S. y Harrison, F. (2004). "The Role of Strategic Planning in the Performance of Small, Professional Service Firms", en Journal of of Management Development, vol.23, pp. 765-776.

Frishmmar, J. (2003). "Information Use in Strategic Decision Making", en Management Decision, 41. 4. $318-326$.

Gemunden, H., Salomo, S. y Krieger, A. (2005). "The influence of project autonomy on project success", en International Journal of Project Management, 23 (2005), pp 366-373.

Gido, J. y Clements, J. P. (1999). Administración Exitosa de Proyectos. México: International Thomson Editores.

Grant, R. M. (1991). "The Resource-Based Theory of Competitive Advantage: Implications for Strategy Formulation", en California Management Review, Spring 33, 3; pp. 114-135.

Grant, R. M. (2010). Contemporary Strategy Analysis. West Sussex - United Kingdom: John Wiley \& Sons, Ltd.

Hahn, W. y Powers, T. L. (2010). "Strategic plan quality, implementation capability, and firm performance", en Academy of Strategic Management Journal, 9(1), 63-81.

Hamel, G. y Prahalad, C. K. (1995). "El Propósito Estrategico", en J. L. Bower, Oficio y Arte de la Gerencia (págs. 90-116). Bogotá D. C.: Editorial Norma S. A.

Herter, G. (1995). "Business planning boosts your chances", en Accounting Technology, vol.11, n. ${ }^{\circ} 4$, pp. 20-26.

I-Ching, L., Seidel, R., Shahbazpour, M. y Howell, D. (2013). "Knowledge management in small and medium-sized enterprises: A New Zealand focus", en Journal of Applied Business Research, 11 (2), 1-21.

IFRS (July de 2013). A review of thr conceptual framework for Financial Reporting. Obtenido de Discussion Paper DP/2013/1: http://www.ifrs. org/current-projects/iasb-projects/conceptual-framework/discussion-paper-july-2013/documents/discussion-paper-conceptual-framework-july-2013.pdf

Infante, A. (1988). Evaluación Financiera de Proyectos de Inversión. Bogotá D.C.: Norma.
Institute, Project Management (2013). A Guide to the Project Management Body of Knowledge. Newtown Square - Pennsylvania: Project Management Instituto, Inc.

Itami, H. (1989). Mobilizing Invisible Assets. Cambridge, MA: Harvard University Press.

Jiménez, A. (2003). "Rutinas organizacionales: un medio para estudiar los cambios recientes en las prácticas de la gerencia estratégica de recursos humanos", en Academia. Revista Latinoamericana de Administración, núm. 30, 2003, pp. 57-86,.

Johannessen, J. A. y Dolva, J. O. (1995). "Innovative Companies' External Information Search in Russia", en International Journal of information Management, 15(5), 367-376.

Johnson, G. y Scholes, K. (2001). Dirección Estratégica. Madrid: Prentice Hall.

Jugdev, K., Mathur, G. y Fung, T. S. (2007). "Project management assets and their relationship with the project management capabilitis of the firm", en International Journal of Project Management, 25, 560-568.

Ketelhohn, W., Marín, J. N. y Montiel, E. L. (2004). Inversiones Análisis de Inversiones Estratégicas. Bogotá: Editorial Norma S.A.

Knight, F. (1921). Risk, Uncertainty and Profit. Boston and New York: The Riverside Press. Knight, F. (1947). Riesgo, incertidumbre y beneficio. Madrid: Aguilar.

Kotler, P. (1999). El Marketing según Kotler. Buenos Aires, Argentina: Editorial Paidos SAICF.

Kubr, M. (2002). La Consultoría de Empresas. México D.F.: Editorial Limusa S.A. de C.V.

Larson, E. W. y F., G. C. (2011). Project Management:The Managerial Process. Singapore: McGraw Hill - International Edition.

Laudon, K. C.y Laudon, J. P. (2014). Management Information Systems. New Jersey: Pearson Higher Education.

López, A. (2005). "La planeación estratégica en la pequeña y mediana empresa: una revisión bibliográfica", en EconoQuantum, vol. 2, n. ${ }^{\circ}$ 1,2005, pp. 141-164. 
López, E. A. (1995). "Macroeconomía Elemental", en Economías para todos (pp. 75 -112). Bogotá D. C.: Fescol.

M., P. y J., P. (1991). "Determinants of Cross-functional Cooperation in the Project Implementation Process", en Project Management Journal, Vol. xxiI; n. ${ }^{\circ} 2 ; 13-20$.

Mahoney, J.y Padian, J. R. (1992). "The Resource-Based View within the Conversation of Strategic Management", en Strategic Management Journal, 13, 363-380.

March, J.y Simon, H. (1994). Teoría de la Organización. Barcelona: Editorial Arial.

Maritan, C. A. (2001). "Capital investment as investing in organizational capabilities: an empirically grounded process model", en Academy of Management Journal, vol. 44 n. ${ }^{\circ}$ 3, 513-531.

Maritan, C. A. y Coen, C. A. (2004). "An Agent-Based Model of Investing in Capabilities: Processes, Decisions and Performance", en Academy of Management Best Papers Proceedings, 1-6.

Mincit (20 de Septiembre de 2014). Ministerio de Comercio Industria y Comercio de Colombia. Obtenido de www.mincit.gov.co/mipymes/descargar. php? id $=40783$

Mintzberg, H. (1973). The Nature of Managerial Work. NJ: Prentice-Hall, Englewood Cliffs.

Mintzberg, H. (1978). "Patterns in Strategy Formation", en Management Science, 24(9), 934-948.

Mintzberg, H. (1992). El poder en la organización. Barcelona: Ariel Economía.

Mintzberg, H., Raisinghani, D. y Théorêt, A. (1976). "The Structure of 'Unstructured' Decision Processes", en Administrative Science Quarterly, vol. 21, n. ${ }^{\circ} 2$, pp. 246-275.

Mokate, K. M. (2007). Evaluación Financiera de Proyectos de Inversión. Bogotá D. C.: Alfaomega.

Morris, M. H., Kuratko, D. F. y Covin, J. G. (2010). Corporate entrepreneurship \& innovation. South-Western Pub.

Müller, R. y Turner, J. R. (2007). "The Influence of Project Managers on Project Success Criteria and Project Success by Type of Project ", en European Management Journal, 25(4), 289 - 309.
Neal, M. y Younis, T. (2006). "Fueling the fire: Professional values and departmental crisis in the UK", en Disaster Prevention and Management, 15(2), 229-312.

Noda, T. y Bower, J. L. (1996). "Strategy making as iterated processes of resource allocation", en Strategic Management Journal, 17(S1): 159-192.

Nonaka, I. (1998). "The Knowledge Creating Company", en H. B. Review, Knowledge Management (pp. 21-45). Boston: Harvard Business School Press. OCED/CEPAL (2012). Perspectivas económicas de América Latina - Políticas de Pymes para el cambio estructural. Obtenido de CEPAL: http://www. cepal.org/publicaciones/xml/4/48374/leo_2013.pdf

Orlandoni, G. (2010). "Escalas de medición en Estadística", en Telos: Revista de Estudios Interdisciplinarios en Ciencias Sociales, 12(2), 243-247.

Ortegón, E., Pacheco, J. F.y Roura, H. (2005). Metodología general de identificación, preparación y evaluación de proyectos de inversión púbilca. Santiago de Chile: ILPES.

Pérez, C. (2009). Técnica de Análisis de Datos con SPSS 15. Madrid: Pearson Educación, S. A.

Pinches, G. E. (1982). "Myopia, Capital Budgeting and Decision Making", en Financial Management, 11, pp 6-19.

Pineda, R. C., Lerner, L. D., Miller, M. C. y Phillips, S. J. (1998). "An investigation of the factors affecting the information-search activities of small business managers", en Journal of Small Business Management, 36(1), 60-71.

Pinto, M. B.y Pinto, J. K. (1991). "Determinants of Cross-functional Cooperation in the Project Implementation Process", en Project Management Journal, vol. XXII; n. ${ }^{\circ} 2 ; 13-20$.

PMI (2013). A Guide to the Project Management Body of Knowledge (known as "Рмвок®"), Fifth Edition. Newtown Square, Pennsylvania: Project Management Institute.

Porter, M. E. (1995). "Cómo forman las estrategias las fuerzas de la competencia", en J. L. Bower, Oficio y Arte de la Gerencia (pp. 37-54). Bogotá D.C.: Editorial Norma S. A. 
Prahalad, C. K. y Hamel, G. (1990). "The Core Competence of the Corporation", en Harvard Business Review, May-June, 79-91.

RAE (2001). Diccionario de la Real Academía de la Lengua Española. España.

Rehman, M., Mahmood, A. K., Sugathan, S. K. y Amin, A. (2010). "Implementation of knowledge management in small and medium enterprises", en Journal of Knowledge Management Practice, 11(1).

Rosillo, J. (2008). Formulación y Evaluación de Proyectos de Inversión. Bogotá D. C.: Cengage Learning Editores S. A.

Rumelt, R. P. (1984). "Toward a strategic theory of firm", en R. Lamb, Competitive strategic management (pp.556-570.). Prentice-Hall.

Sahin, C. (2001). "Managing profesional service firms: role of managerial abilities", en Journal of Management Development, 30(4); 413-426.

Sapag, N. (2001). Evaluación de Proyectos de Inversión en la Empresa. Buenos Aires: Pearson Education S.A.

Sapag, N.y Sapag, R. (2008). Preparación y Evaluación de Proyectos. México D.F.: McGrawHiil Interamericana S.A.

Schmid, B.y Adams, J. (2008). "Motivation in project management: The project manager's perspective", en Project Management Journal, 39(2), 60-71.

Seglelod, E. (1998). "Capital budgeting in a fast changing world", en Long Range Planning, 31(4) 529-541.

Simon, H. (1972). El Comportamiento Administrativo. Madrid: Ediciones Aguilar S.A.

Simon, H. A. (1965). The Shape of Automation. New York: Harper and Row.
Stevens, S. (1946). "On the Theory of Scales of Measurement", en American Association for the Advancement of Science, vol. 103, n. ${ }^{\circ} 2684$, pp. 677-680.

Stevens, S. (1957). "On the Psychological Law", en Psychological Review, 64, pp. 153-181. American Psychological Association. USA.

Takeuchi, I. (2001). "Towards a Universal Management Concept of Knowledge", en I. Nonaka y D. Teece, Managing Industrial Knowledge (págs. 315-329). London: Sage Publications.

Thomas, J.y Mullaly, M. (2007). "Understanding the value of project management: First steps on an international investigation in search of value", en Project Management Journal, 38(3):74-90.

V.L., C. y Crittenden, W. (2008). Building a capable organization: The eight levers.

Van Horne, J. C. (1997). Administración Financiera. México D. F.: Pearson Educación.

Wernerfelt, B. (1984). "A resource-based view of the firm", en Strategic Management Journal, 5(2), 171-80.

Wernerfelt, B. (1989). "From Critical Resources to Corporate Strategy", en Journal of General Management, vol. 14, pp. 4-12.

Wernerfelt, B. (1995). "The Resource-based View of the Firm. Strategic Management Journal", en Strategic Management Journal, vol.16, n. ${ }^{\circ} 13$, 171-174.

Wheelwright, S. A. (1992). Revolutionizing Product Development: Quantum Leaps in Speed, Efficiency, and Quality. New York, NY: The Free Press.

Wuellner, W. (1990). "Project Performance Evaluation Checklist for Consulting Engineers", en Journal of Management in Engineering, 6; 3; 270-281. 